\title{
Neuroprotective effect of combining tanshinone IIA with low-dose methylprednisolone following acute spinal cord injury in rats
}

\author{
NIAN-WEI YAO ${ }^{1-3^{*}}$, YUAN LU $^{4 *}$, LI-QI SHI ${ }^{5}$, FENG XU ${ }^{2}$ and XIAN-HUA CAI ${ }^{2}$ \\ ${ }^{1}$ Department of Orthopedics, The Third Affiliated Hospital of Nanjing University of Traditional Chinese Medicine, \\ Nanjing Hospital of Traditional Chinese Medicine, Nanjing, Jiangsu 210000; ${ }^{2}$ Department of Orthopedics, \\ Wuhan General Hospital of Guangzhou Military Command, Wuhan, Hubei $430070 ;{ }^{3}$ College of Acupuncture and Orthopedics, \\ Hubei University of Traditional Chinese Medicine, Wuhan, Hubei 430065; ${ }^{4}$ Department of Neurology, \\ Nantong First People's Hospital, Nantong, Jiangsu 226000; ${ }^{5}$ Department of Orthopedics, \\ Yuyao Hospital of Traditional Chinese Medicine, Ningbo, Zhejiang 315000, P.R. China
}

Received September 5, 2015; Accepted January 13, 2017

DOI: 10.3892/etm.2017.4271

\begin{abstract}
The present study compared the potential neuroprotective effect of tanshinone IIA (TIIA) monotherapy, methylprednisolone (MP) monotherapy and combined treatment in an adult acute spinal cord injury (ASCI) rat model. The current study used the weight-drop method (Allen's Impactor) in the rat model and the mechanical scratch method in primary spinal cord neuron culture to determine whether the combined treatment was able to reduce the required dosage of MP in the treatment of ASCI to produce a similar or improved therapeutic effect. In vivo male Sprague Dawley rats $(n=60)$ were randomly divided into 5 groups, of which 12 rats were selected for the sham group and T9-T11 laminectomies, leading to ASCI, were performed on 48 of the 60 rats using a $10 \mathrm{~g} \mathrm{x} 25 \mathrm{~mm}$ weight-drop at the level of T10 spinal cord. Therefore, the ASCI group ( $\mathrm{n}=12)$ included the 'laminectomy and weight-drop'. The remaining 36 ASCI model animals were subdivided into 3 groups ( $\mathrm{n}=12$ each group): TIIA group (30 mg/kg/day), MP group (30 mg/kg) and combined treatment group (TIIA $30 \mathrm{mg} / \mathrm{kg} / \mathrm{day}+$ MP $20 \mathrm{mg} / \mathrm{kg}$ ). Neuronal function following ASCI was evaluated using the Basso Beattie Bresnahan (BBB) locomotor rating scale. Levels of the anti-apoptotic factor B-cell lymphoma-2 (Bcl-2), the pro-apoptotic factors $\mathrm{Bcl}-2$ associated protein $\mathrm{X}$ (Bax) and caspase-3, and the inflammatory associated factor nuclear factor- $\mathrm{\kappa} \mathrm{B}$, were analyzed by western blot analysis. Immunohistochemistry was used to detect caspase-3. To investigate the underlying
\end{abstract}

Correspondence to: Professor Xian-Hua Cai, Department of Orthopedics, Wuhan General Hospital of Guangzhou Military Command, 627 Wuluo Road, Wuhan, Hubei 430070, P.R. China E-mail:whzyycaixh@sina.com

*Contributed equally

Key words: tanshinone IIA, methylprednisolone, acute spinal cord injury, apoptosis factors, anti-inflammatory, anti-apoptotic mechanism, the anti-oxidative effect of combination TIIA and MP treatment was assessed by measuring the activity of malondialdehyde (MDA) and superoxide dismutase (SOD) in ASCI. In agreement with the experiment in vivo, primary neurons were prepared from the spinal cord of one-day-old Sprague-Dawley rats' and co-cultured with astrocytes from the brain cortex. The injury of neurons was induced by mechanical scratch and levels of apoptosis factors were analyzed by western blot analysis. The results of the current study indicated that injured animals in the combined treatment group exhibited a significant increase in BBB scores $(\mathrm{P}<0.05)$. TIIA + MP combined treatment and MP treatment was observed to reduce the expression of pro-apoptotic factors and promote neuron survival in vivo and in vitro. Combined treatment may promote neuroprotection through reduced apoptosis and inflammation caused by ASCI, similar to MP alone. Combined treatment reversed the decrease of SOD and the increase of MDA level caused by ASCI. In addition, combined treatment decreased the expression of caspase- 3 in the neurons following ASCI in rats, as indicated by immunofluorescence double labeling. Overall, the present study indicates that the combined treatment of TIIA and MP may protect the neurons by stimulating the rapid initiation of neuroprotection following ASCI and reduce the dosage of MP in the treatment of ASCI required to produce the same or improved neuroprotective effects in vivo and in vitro.

\section{Introduction}

Acute spinal cord injury (ASCI) is a severe type of high-energy central nervous system (CNS) damage that frequently leads to devastating neurological deficits and disabilities, including the loss of sensory and motor capabilities (paraplegia or tetraplegia). The prevalence of ASCI ranges from 906 to 250 per million and the annual incidence of ASCI ranges from 8 to 49.1 per million in different nations (1) and global prevalence is expected to increase (2). Following ASCI, the spinal cord is restored for a period of time, however function is markedly damaged and is often irreversible, leading to delayed death of spinal neurons and paralysis. There is an increasing body 
of evidence regarding the pathophysiology of acute spinal cord injury, indicating that it involves primary and secondary injury (3-10). The primary injury directly leads to neuron death, while the secondary injury's pathological mechanisms encompass excitotoxicity, ionic imbalances, inflammatory response, lipid peroxidation, free radical injury and apoptosis (3-7), which induce neuron death. Presently, stem cell therapy has become one of the current treatments; however, its application is limited due to ethical aspects (11). Although great advances in pharmacotherapy for the purpose of limiting neuronal secondary injury following ASCI have been achieved, only methylprednisolone (MP) is used widely $(9,12,13)$. MP is used to treat ASCI due to its potent anti-inflammatory, anti-apoptosis and antioxidant properties (9). However, due to the poor side-effects, such as sepsis, pulmonary embolism, wound infections (14) and gastrointestinal bleeding (2), and controversy over whether or not the effects are beneficial-previous studies have reported that MP did not improve motor function scores $(2,15)$-concerns have been raised due to the high dosage (30 mg/kg bolus followed by $5.4 \mathrm{mg} / \mathrm{kg}$ for $23 \mathrm{~h}$ ) (13) required and therefore, its use in treating ASCI is contentious $(9,16)$. It is therefore important to investigate other pharmacological agents that may be combined with MP in order to reduce the dose of MP required and enhance functional recovery.

Tanshinone IIA (TIIA) is an effective monomer component purified from the Chinese herb Danshen (Radix Salviae Miltiorrhiza) and representative liposoluble constituent. It participates in various biochemical events in organisms and exhibits bioactivities to antagonize oxidation properties, protect against inflammation, apoptosis and free radical injury following CNS injury, as demonstrated in in vitro and in vivo studies $(8,17,18)$. It has been demonstrated that TIIA protects rat spinal cord neurons against ASCI-induced neurotoxicity (8). However, to the best of our knowledge, there are no studies assessing the effect of combined TIIA with MP and whether combining TIIA treatment with MP may allow the dose of MP to be reduced in the treatment of ASCI. The present study attempted to investigate the effects of combined TIIA with MP to treat ASCI in vivo and in vitro by measuring oxidative stress reaction, the expressions of inflammatory factors and apoptosis-related proteins.

\section{Materials and methods}

Animals. All Sprague-Dawley rats were kept in dams in the Laboratory Animal Services Center with ad libitum access to food and water and a 12-h light-dark cycle. All rats were maintained at $22-25^{\circ} \mathrm{C}$ and $40-60 \%$ humidity. A total of 60 adult male Sprague-Dawley rats (Hubei Provincial Laboratory Animal Public Service Center, Hubei, China), weighing 240-260 $\mathrm{g}$ and aged 6-7 weeks-old, were used in the current study, 12 of which were selected for sham-surgery and 48 selected for contusive ASCI. A total of 80 one-day-old male Sprague-Dawley rats (Center for Animal Experiment of Wuhan University, Wuhan, China) were used for cell culture in vitro. All rats were maintained in the same conditions. All procedures were performed in accordance with guidelines approved by the Animal Ethics Committee of Wuhan General Hospital of Guangzhou Military Command (Wuhan, China).
Rat ASCI model. All adult animals $(\mathrm{n}=60)$ were anaesthetized through intraperitoneal (i.p.) injection of $10 \%$ chloral hydrate (3.5 ml/kg, i.p.; Hubei Yuancheng Saichuang Technology Co., Ltd., Wuhan, China). In order to expose the paravertebral muscles, an incision was made along the back of each rat. A laminectomy was performed at the T9-T11 level, exposing the cord without damaging the dura. In order to subject the exposed dorsal surface of the cord to the weight drop impact, a 10-g metal rod was dropped from a height of $25 \mathrm{~mm}$ (19). Subsequently, the lesioned muscle and skin were sutured in two layers. Each rat was then administered $1.0 \mathrm{ml}$ saline solution using a subcutaneous injection, to replace blood volume lost during surgery. Following surgery, sterile gauze was wrapped around the wound on each rat. A standard diet and water were provided daily to the rats ad libitum. A total of 3 to 5 days' post-injury, manual bladder expression was performed twice a day until the rats were able to urinate by themselves.

Study design in animal models. In the current study, 60 rats were randomly assigned into five groups $(n=12$, each group) and all animals were subjected to laminectomy: Sham group, animals were only subjected to laminectomy; ASCI group, animals subjected to ASCI using an impactor; TIIA treatment group, animals subjected to ASCI using an impactor and treated with a dosage of $30 \mathrm{mg} / \mathrm{kg}$ TIIA per day [chosen according to previous findings (20)]; MP treatment group, animals subjected to ASCI using an impactor and treated with $30 \mathrm{mg} / \mathrm{kg}$ MP (13) $15 \mathrm{~min}$ following the strike; TIIA + MP treatment group, the animals were subjected to ASCI using an impactor and treated with $30 \mathrm{mg} / \mathrm{kg}$ TIIA per day and a selected low dose of $20 \mathrm{mg} / \mathrm{kg}$ MP $15 \mathrm{~min}$ after the strike. The drugs were administered via intraperitoneal administration. At the planed time point, rats were anesthetized by intraperitoneal injection of $10 \%$ chloral hydrate $(3.5 \mathrm{ml} / \mathrm{kg}$, i.p.) as before and the spinal cord was immediately exposed from $\mathrm{T} 9$ to T11 and $10 \mathrm{~mm}$ damaged tissue was cut at the site of injury. Rats were sacrificed by cervical dislocation except for the immunofluorescence double labeling experiment at different time points (4 rats per group), depending on the parameters measured: Rats undergoing western blot analysis for nuclear factor (NF)- $\kappa \mathrm{B}$ on day 1, measurement of tissue superoxide dismutase (SOD) and malondialdehyde (MDA) on day 1 and on day 7 following ASCI, immunofluorescent staining on day 3 following ASCI and western blot analysis for caspase-3, B-cell lymphoma-2 (Bcl-2) and Bcl-2 associated protein $\mathrm{X}$ (Bax) on day 7 following ASCI.

Cell culture and treatment. Primary culture of cortical neurons was isolated and cultured as previously described (21), with minor modifications. For mixed glial cell culture, one-day-old Sprague-Dawley rat pups were sacrificed by cervical dislocation; the skin and skull were carefully torn with tweezers and brain cortical tissues were dissected in ice-cold dissection buffer. Following this, the meninges of the cortex were peeled off and separated from the olfactory bulb and hippocampus and dissected cortical tissues were digested with trypsin. Following incubation at $37^{\circ} \mathrm{C}$ for $15 \mathrm{~min}$ to adhere and eliminate fibroblasts, cells were dissociated and plated into $75 \mathrm{~cm}^{2}$ tissue culture flasks at a density of $2.0 \times 10^{7}$ cells/flask. Cells were incubated at $37^{\circ} \mathrm{C}$ with Dulbecco's modified Eagle's 
medium/F12 supplemented with $10 \%$ fetal bovine serum (both Hyclone; GE Healthcare Life Sciences, Logan, UT, USA) and $1 \%$ penicillin-streptomycin medium. Following $24 \mathrm{~h}$, non-adherent cells were removed by agitating the flask and the culture medium was replaced. When a confluent layer of glial cells formed (10 to 14 days), cells were trypsinized (Beyotime Institute of Biotechnology, Haimen, China) and inoculated. Following the second passage, cells were seeded into a 6-well cell culture plate (Nest Biotechnology Co., Ltd., Wuxi, China) for neuron-glia co-culture. Rat primary spinal cord neuron cultures were derived from the spinal cord of one-day-old Sprague-Dawley rat pups. Briefly, spinal cords were dissected and freed of meninges. Cells were dissociated by trypsinization, followed by triturating and passing through a $74-\mathrm{mm}$ steel mesh. Cells were incubated at $37^{\circ} \mathrm{C}$ for $30 \mathrm{~min}$ to allow adherence and eliminate glial cells and fibroblasts. Neurons $\left(2 \times 10^{5}\right.$ cells/plate) were plated onto poly-L-lysine (PLL; Sigma-Aldrich; Merck Millipore, Darmstadt, Germany) coated 20-mm glass coverslips (NEST, China) for co-culture experiments and MTT analysis. Neurons were maintained in neurobasal media (Gibco; Thermo Fisher Scientific, Inc., Waltham, MA, USA), 2\% B27 supplement (Gibco; Thermo Fisher Scientific, Inc.) and $0.5 \mathrm{mM}$ glutamine (Sigma-Aldrich; Merck Millipore). Neurons on coverslips at day 6-7 were placed into glia-seeded wells. Neurons and astrocytes were in close apposition but with no direct cell-cell contact. For modelling ASCI, neurons and glial cell were subjected to a cut with a no. 15 scalpel blade (22) with a $1 \mathrm{~mm}$ gap. Cultures were maintained in a $37^{\circ} \mathrm{C}$ humidified incubator in a $5 \% \mathrm{CO}_{2}$ atmosphere. The cells were treated with TIIA at a concentration of $10 \mu \mathrm{M}$ (according to the MTT analysis), MP at a concentration of $1 \mu \mathrm{M}$ and combined TIIA $10 \mu \mathrm{M}$ and $\mathrm{MP}$ $0.5 \mu \mathrm{M}$, respectively. The incubation was continued for $24 \mathrm{~h}$ and subsequently the cells were trypsinized, collected and extracted for analysis.

MTT analysis. The neurons' viability was measured by MTT assay for the optimal concentration of TIIA for neurons. Briefly, the MTT solution (Beyotime Institute of Biotechnology) was added to 96 -well plates $\left(1 \times 10^{4}\right.$ neuronal cells/well) at a final concentration of $500 \mathrm{mg} / \mathrm{ml}$ and then incubated for $4 \mathrm{~h}$ at $37^{\circ} \mathrm{C}$. To dissolve the formazan, the culture medium was removed and $100 \mu$ dimethyl sulfoxide was added to each well. The optical density of each well was measured at $570 \mathrm{~nm}$ using a microplate reader. One culture well with neurons was used for each experimental condition. Thus, each plate contained multiple wells of each experimental condition and multiple control wells. This procedure was replicated for 4 plates per condition. Spinal cord neurons were treated with various concentrations $(0,0.1,0.5,1$, $5,10,30,50,70$ and $90 \mu \mathrm{M})$ of TIIA for $24 \mathrm{~h}$. Following this, the cells were injured as previously described. Cell viability was measured using an MTT assay. Neuron viability treated with TIIA, MP and their combination was measured by MTT assay too. The MTT data was converted to the percentage of the respective control groups (untreated spinal cord neuron cells) after analysis by microplate reader (SM-600, Shanghai Utrao Medical Devices Co., Ltd, China).

Western blot analysis. Total proteins were extracted separately from the spinal cord tissues or neurons' cultures. In detail, following sacrifice and transcardial perfusion with $100 \mathrm{ml}$ $0.9 \%$ saline, the experimental rats' skin and soft tissue on back at T9-T11 were cut to expose the injured spinal cord, which was harvested $(5 \mathrm{~mm}$ rostral and $5 \mathrm{~mm}$ caudal from the injury site). The tissue was homogenized by sonication in radioimmunoprecipitation assay lysis buffer (Wuhan Boster Biological Technology, Ltd., Wuhan, China), and neurons were washed three times in Earle's Balance Salt Solution following trypsinization and kept in serum-free media (Hyclone; GE Healthcare Life Sciences). The samples were centrifuged at 12,000 x $g$ for $1 \mathrm{~h}$ at $4^{\circ} \mathrm{C}$. The protein content of the lysate samples was determined using a BCA protein assay kit (Beyotime Institute of Biotechnology). Protein lysates ( $35 \mu \mathrm{g}$ per lane for each sample) were fractioned using $10 \%$ SDS-PAGE and transferred to a nitrocellulose membrane. Membranes were then blocked with $5 \%$ non-fat dry milk at room temperature for $2 \mathrm{~h}$ and incubated for $12 \mathrm{~h}$ at $4^{\circ} \mathrm{C}$ with primary antibodies: Rabbit anti- $\beta$-actin (BA2305; 1:1,000; Wuhan Boster Biological Technology, Ltd.), rabbit anti-Bcl-2 (15071T; 1:800), rabbit anti-Bax (2772T; 1:800; both Cell Signaling Technology, Inc., Danvers, MA, USA), rabbit anti-caspase-3 (ab4051; 1:300; Abcam, Cambridge, UK) and rabbit anti-NF-кBP65 (BA1298; 1:1,000; Wuhan Boster Biological Technology, Ltd.). Horseradish peroxidase conjugate goat anti-rabbit immunoglobulin (Ig)G was used as a secondary antibody (BA1055 1:50,000, Wuhan Boster Biological Technology, Ltd.). The reaction was developed using enhanced chemiluminescence (ELC) reagent (Thermo Fisher Scientific, Inc.) and signal density was measured using ImageJ analysis software v2.1.4.7 (National Institute of Health, Bethesda, MD, USA) (23). Each group had 4 replicates and the values were normalized against $\beta$-actin.

Immunofluorescence double labeling. In order to perform immunofluorescence double labeling for confocal microscopy, rats were sacrificed 3 days post-injury as described before, and the whole body of the rat was fixed using transcardial saline infusion followed by $100 \mathrm{ml}$ paraformaldehyde (4\%). Following perfusion, the injured spinal cords were carefully dissected, as indicated for western blot analysis, fixed for an additional $2 \mathrm{~h}$ in $4 \%$ paraformaldehyde at $4{ }^{\circ} \mathrm{C}$. The specimens were transferred to a solution containing $30 \%$ sucrose in $0.1 \mathrm{M}$ phosphate buffer ( $\mathrm{pH}$ 7.4) overnight. Spinal cord segments from sham-operated or injured animals were embedded in paraffin and longitudinally sectioned (2- $\mu \mathrm{m}$ thick), prior to mounting on gelatin-coated slides. The sections were dewaxed with xylene, permeabilized and blocked with $0.3 \%$ Triton X-100 and 10\% normal goat serum (Wuhan Boster Biological Technology, Ltd.) in 0.01 M phosphate-buffered saline for $30 \mathrm{~min}$ at $4^{\circ} \mathrm{C}$. Mouse anti rat caspase-3 polyclonal antibody (AB208161; 1:100; Abcam) and rabbit anti-rat $\beta$-III Tubulin (AT809; 1:100; Beyotime Institute of Biotechnology) primary antibodies were applied to the sections overnight at $4^{\circ} \mathrm{C}$. On the following day, the sections were incubated with fluorescein isothiocyanate-conjugated goat anti-rabbit (BA1105; 1:1,000) and indoles cyanine dye (Cy3)-conjugated goat anti-mouse (BA1032; 1:1,000; both Wuhan Boster Biological Technology, Ltd.) secondary antibodies at $4^{\circ} \mathrm{C}$ for $3 \mathrm{~h}$. Slides were mounted and examined with a BX51 fluorescence microscope (Olympus Corp., Tokyo, Japan). In control sections, the primary antibody 
was substituted with $1 \%$ normal goat serum. The colocalisation area was measured using ImageJ analysis software (v2.1.4.7). $\mathrm{R}$ value represented Mander's overlap coefficient tubulin and caspase-3 in Image J analysis software: Ranges between 1 and 0 , with 1 indicating high colocalisation and 0 indicating low colocalisation.

Behavioral assessments. Behavioral assessments were determined using the Basso, Beattie, and Bresnahan (BBB) locomotor rating score. Gross $\mathrm{BBB}$ locomotor recovery following contusive spinal cord injury was scored in an open field according to the locomotor rating scale of 0 (complete paralysis) to 21 (normal locomotion) (24). BBB testing was performed once a day in double-blind model from day 0 to day 7 following spinal cord surgery. Each rat was observed for 4 min by two investigators blinded to the study.

Measurement of SOD and MDA. MDA was measured using an MDA assay kit (Thiobar bituric acid test method; Beyotime Institute of Biotechnology) and SOD activities in spinal cord tissues were measured using a Total-SOD (T-SOD) assay kit (Hydroxylamine method; Nanjing Jiancheng Bioengineering Institute, Nanjing, China) in accordance with previous research (20).

Statistical analysis. Data are expressed as the mean \pm standard error of the mean. Statistical significance was determined using Student's t-test when there were two experimental groups. When more than two groups were compared, statistical evaluation of the data was performed using one-way analysis of variance and Dunnett's post-hoc test. $\mathrm{P}<0.05$ was considered to represent a statistically significant difference.

\section{Results}

Effects on functional recovery following ASCI. In order to assess the effects of treatment on functional recovery following ASCI in each group, the BBB locomotor test was performed every day for 7 days (Fig. 1). On day 0, the BBB score of all rats was regarded as 0 . BBB scores in the treatment groups were significantly higher than the ASCI group on day 3 and 7 $(\mathrm{P}<0.05)$. BBB scores in the TIIA + MP combined group were higher than the MP group on day 3, but this was not significant $(\mathrm{P}>0.05)$. From day 5 to day 7 , the scores in the combined treatment group were higher than the MP group and the differences were statistically significant $(\mathrm{P}<0.05$; Fig. 1$)$.

Effect on the expression of apoptosis factors following ASCI. In the present study, the effect of each treatment on the expression of pro- and anti-apoptotic factors on day 7 following ASCI in rats and $24 \mathrm{~h}$ following mechanical injury in neurons were investigated using western blot analysis. As presented in Fig. 2A, Bax and caspase-3 expression were appreciably increased in the spinal cords of rats subjected to ASCI and Bcl-2 expression was decreased compared with the sham group. However, this state was altered by the drug treatments. Combined treatment exhibited significant anti-apoptotic ability compared with that observed in the ASCI group on day 7 after ASCI $(\mathrm{P}<0.05$; Fig. 2A). The statistical analysis demonstrated that $\mathrm{Bcl}-2$ expression in the combined treatment group was significantly increased, however, levels of Bax and caspase-3 were significantly decreased compared with the MP group ( $\mathrm{P}<0.05$; Fig. 2A). In addition, the data in Fig. 2B indicated that combined treatment was significantly more effective than TIIA treatment alone at reversing the decrease in Bcl-2 expression induced by mechanical injury in neurons $(\mathrm{P}<0.05)$, although Bax and caspase-3 expression did not differ significantly. These effects were similar to those observed in the MP group $(\mathrm{P}<0.05)$.

Effect on the expression of antioxidation molecules following $A S C I$. The effect of each treatment on antioxidation was investigated by measuring the tissue levels of SOD and MDA in the spinal cord on day 1 and day 7. Levels of SOD were significantly elevated in the MP group and TIIA + MP group compared with the ASCI group ( $\mathrm{P}<0.05$; Fig. 3A), while levels of MDA were significantly reduced in the MP group and TIIA + MP group compared with those of the rats in the ASCI group $(\mathrm{P}<0.05$; Fig. 3B). However, no significant differences in SOD and MDA levels were observed between the MP and combined treatment groups.

Effect on the expression level of $N F-\kappa B$ in the spinal cord following ASCI. The effect of each treatment on $\mathrm{NF}-\kappa \mathrm{B}$ expression in the spinal cord tissue on 1 day following ASCI was detected by western blot analysis. This was due to the $\mathrm{NF}-\kappa \mathrm{B}$ associated pathway that serves a key role in inflammation during secondary injury following ASCI (25). Substantial increases in NF- $\mathrm{NB}$ levels were identified in spinal cord tissue samples collected from rats 1 day following ASCI. These increases were significantly reversed in all treatment groups $(\mathrm{P}<0.05$; Fig. 4). NF- $\kappa \mathrm{B}$ levels detected in the combined treatment group were lower than in the MP group, but this difference was not significant.

Effect on the activity of mechanical injured neurons following ASCI. To determine the neuroprotective ability of TIIA, cultured spinal cord neurons were exposed to mechanical injury and cell viability was assessed by MTT reduction assay after $24 \mathrm{~h}$. Results are presented as the mean of 5 replicate values in three independent experiments. Based on the result, $10 \mu \mathrm{M}$ was selected as the optimal TIIA concentration for subsequent experiments, as the increase in neuronal activity was greatest following treatment with $10 \mu \mathrm{M}$ TIIA (Fig. 5A). In the ASCI group, the optical density (OD) value decreased $24 \mathrm{~h}$ after the mechanical scratch protocol. Treatment with TIIA, MP and the combined treatment significantly reversed this decrease in $\mathrm{OD}$ value $(\mathrm{P}<0.05)$. Cell viability in the combined treatment group was higher than in the MP group, however, no significant differences were observed in cell viability between cells treated with MP alone and the combined treatment.

Effect on the expression of caspase-3 in spinal tissues and neurons in vivo by immunohistochemistry. Immunofluorescence double labeling images are presented in Fig. 6A (red: Tubulin and green: caspase-3). The results indicated that decreased levels of caspase-3 expression in neurons were exhibited in treatment groups when compared with the ASCI group. In Fig. 6B, the R values represent the overlap coefficient of tubulin and caspase- 3 


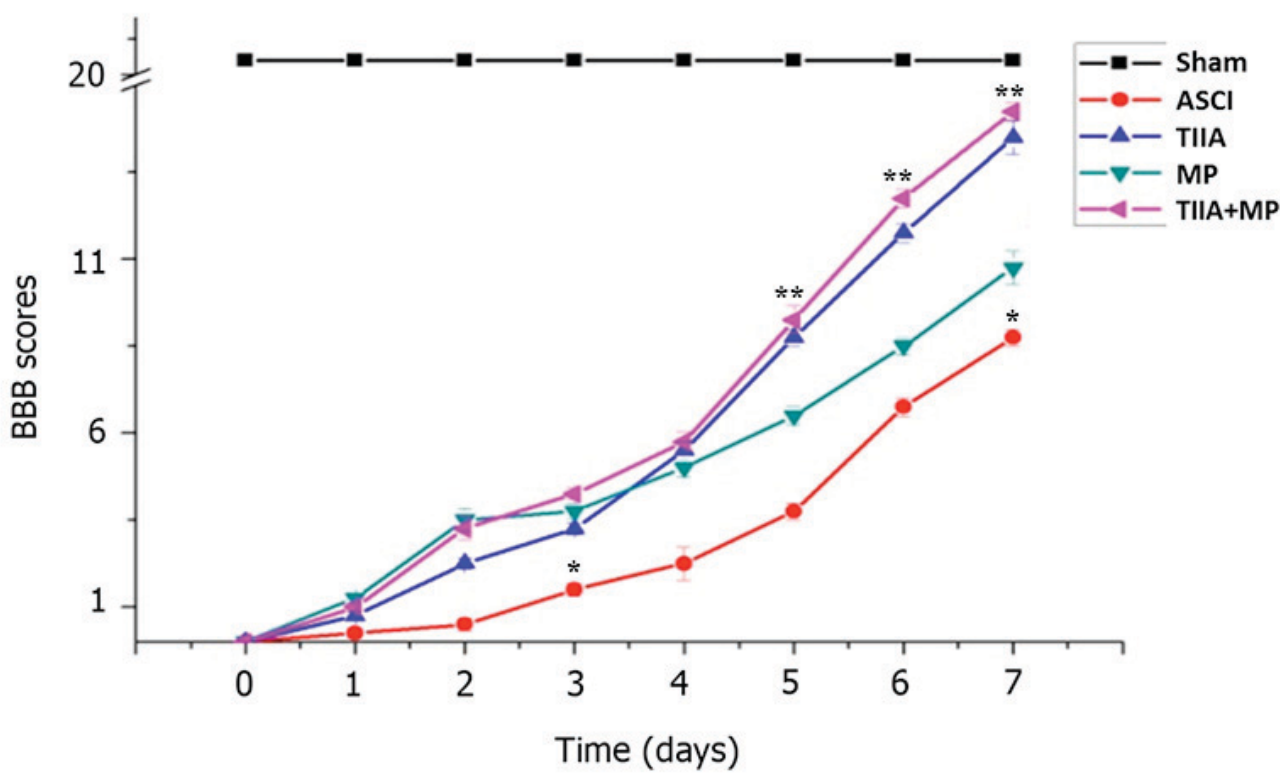

Figure 1. Effects of each group on functional recovery following ASCI by BBB scores. ${ }^{*} \mathrm{P}<0.05$ vs. all treatment groups; ${ }^{* *} \mathrm{P}<0.05$ vs. MP group. ASCI, acute spinal cord injury; BBB, Basso Beattie Bresnahan; MP, methylprednisolone; TIIA, tanshinone IIA; TIIA + MP, TIIA and MP combined treatment; Sham, sham-operated, no treatment.
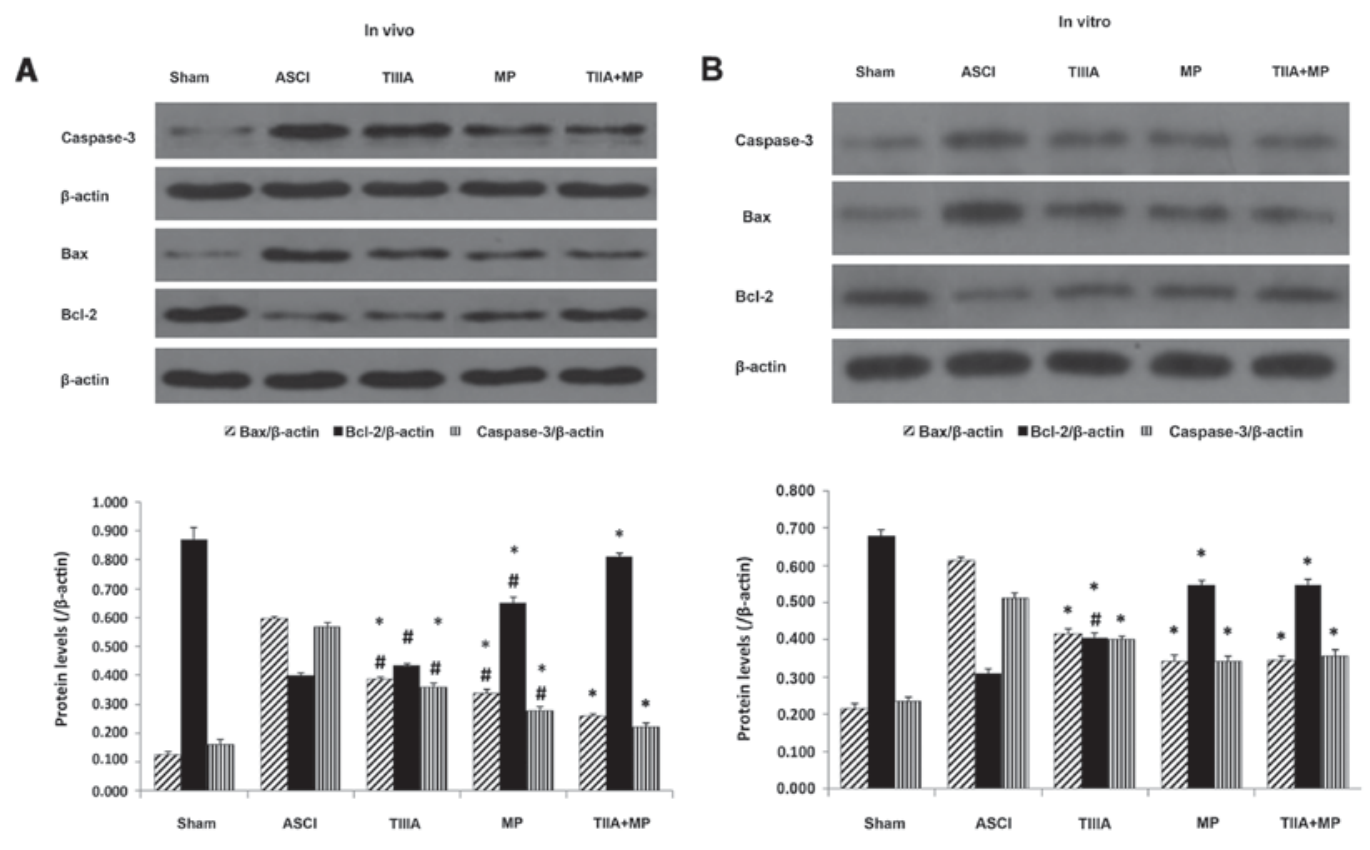

Figure 2. Expression of Bcl-2, Bax and Caspase-3 proteins determined by western blot analysis. (A) Results normalized relative to $\beta$-actin in the ASCI rats at day 7 and (B) in spinal neuron culture scratch models following $24 \mathrm{~h}$ culture. Data are presented as mean \pm standard error of the mean, $\mathrm{n}=4$ per group. (A) "P<0.05 vs. ACSI group; ${ }^{*} \mathrm{P}<0.05$ vs. TIIA + MP group. (B) "P $<0.05$ vs. ASCI group; ${ }^{\text {" }} \mathrm{P}<0.05$ vs. TIIA + MP group and MP group. Bcl-2, B-cell lymphoma-2; Bax, Bcl-2 associated protein X; ASCI, acute spinal cord injury; MP, methylprednisolone; TIIA, tanshinone IIA; TIIA + MP, TIIA and MP combined treatment; Sham, sham-operated, no treatment.

and the results from the immunohistochemistry experiments revealed that a significant increase in the immunoreactivity of caspase-3 was observed in ASCI rats compared with the sham group $(\mathrm{P}<0.05)$, which suggested that there was more caspase-3 present in neurons in the ASCI group compared with the sham group. Similar to the results from western blot analysis, TIIA,
MP and combined TIIA + MP treatment significantly reduced the $\mathrm{R}$ value compared with the ASCI group, which indicated that reduced levels of caspase-3 $(\mathrm{P}<0.05)$ were present in the neurons of each group, but there was no significant difference between the TIIA or MP groups compared with the combined treatment group $(\mathrm{P}>0.05)$. 
A

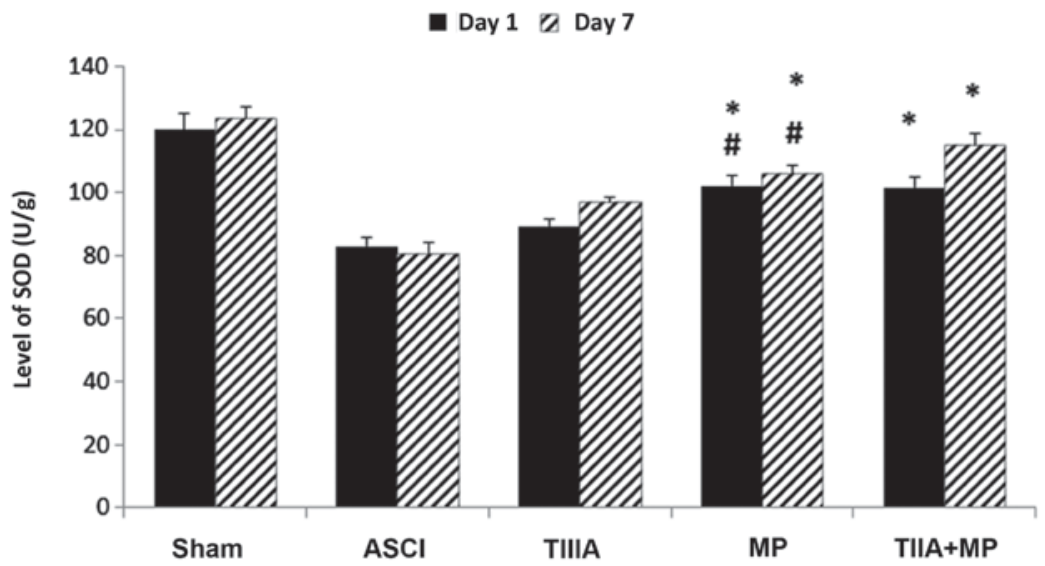

B

- Day 1 Day 7

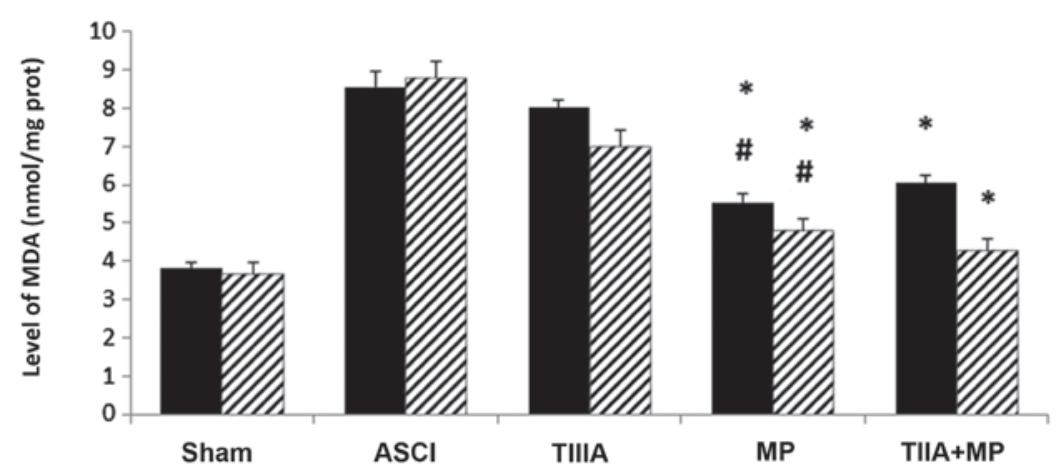

Figure 3. Expression of antioxidation molecules. The expression of (A) SOD and (B) MDA were determined at day 1 and 7 in ASCI rats. Data are presented as mean \pm standard error of the mean, $\mathrm{n}=4$ per group. (A) " $\mathrm{P}<0.05$ vs. ASCI group; ${ }^{~} \mathrm{P}>0.05$ vs. TIIA $+\mathrm{MP}$ group. (B) " $\mathrm{P}<0.05$ vs. ASCI group; ${ }^{*} \mathrm{P}>0.05$ vs. TIIA + MP group. SOD, superoxide dismutase; MDA, malondialdehyde; ASCI, acute spinal cord injury; MP, methylprednisolone; TIIA, tanshinone IIA; TIIA + MP, TIIA and MP combined treatment; Sham, sham-operated, no treatment.

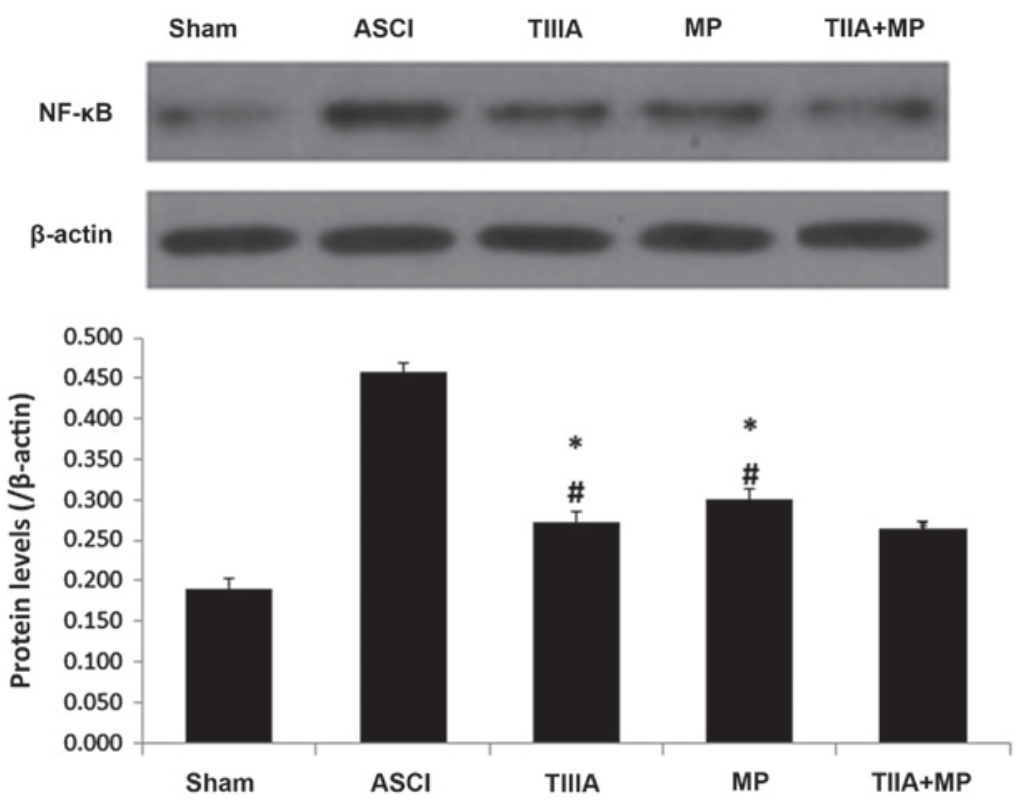

Figure 4. Expression of NF- $\mathrm{BB}$ determined by western blot analysis, with results normalized relative to $\beta$-actin in spinal neurons culture scratch models following $24 \mathrm{~h}$. Data are presented as mean \pm standard error of the mean, $\mathrm{n}=4$ per group. ${ }^{*} \mathrm{P}<0.05$ vs. ASCI group, ${ }^{*} \mathrm{P}>0.05$ vs. TIIA $+\mathrm{MP}$ group. NF- $\mathrm{kB}$, nuclear factor- $\mathrm{kB}$; ASCI, acute spinal cord injury; MP, methylprednisolone; TIIA, tanshinone IIA; TIIA + MP, TIIA and MP combined treatment; Sham, sham-operated, no treatment. 
A

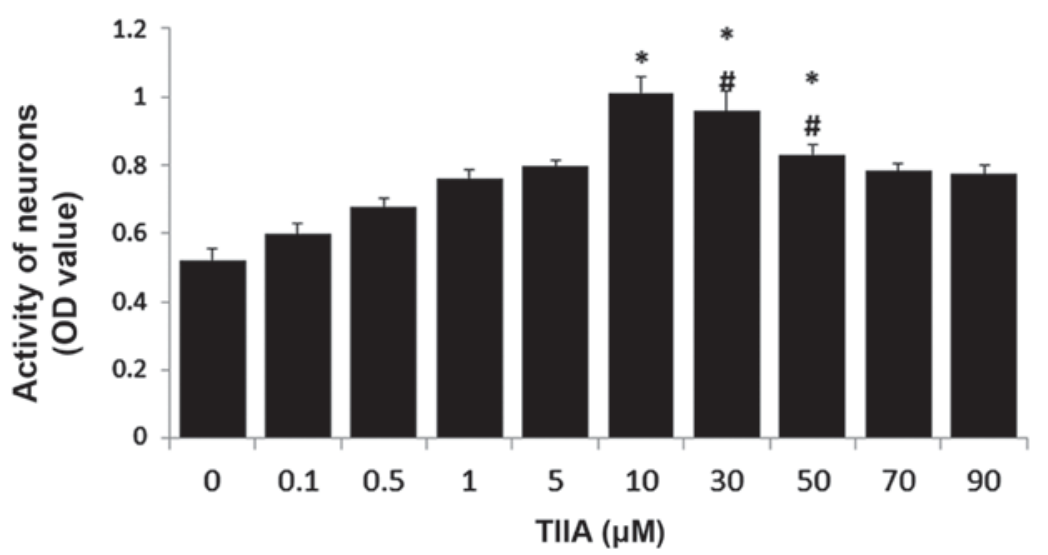

B

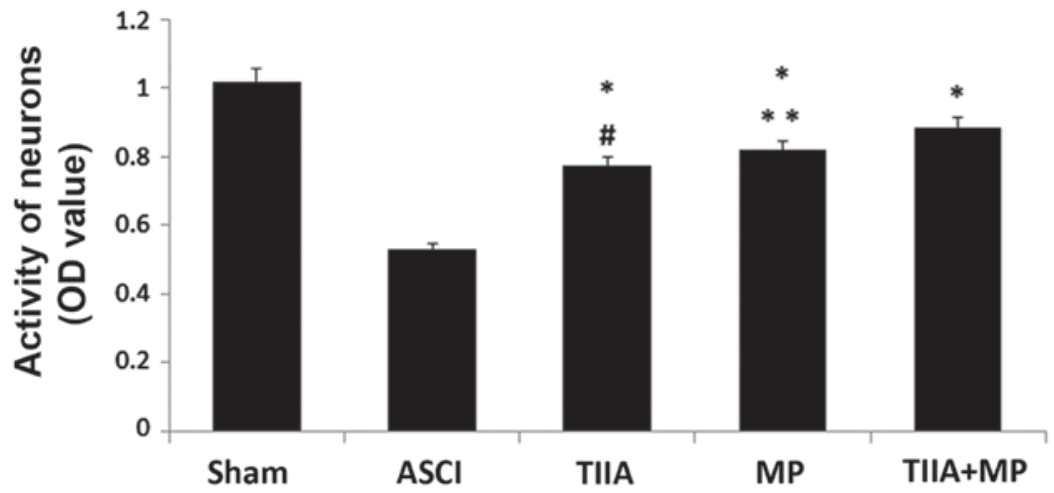

Figure 5. Activity of neurons to assess and select the concentration of TIIA required for subsequent experiments. (A) ${ }^{*} \mathrm{P}<0.05$ vs. unmarked groups; ${ }^{\#} \mathrm{P}>0.05$ vs. $10 \mu \mathrm{M}$ group. (B) ${ }^{*} \mathrm{P}<0.05$ vs. ASCI group; ${ }^{\#} \mathrm{P}<0.05$ vs. TIIA + MP group; ${ }^{* *} \mathrm{P}>0.05$ vs. TIIA + MP group. TIIA, tanshinone IIA; ASCI, acute spinal cord injury; MP, methylprednisolone; TIIA + MP, TIIA and MP combined treatment; OD, optical density.

\section{Discussion}

The present study provides evidence that the combination of TIIA with MP significantly improves motor function following ASCI and may reduce the dose of MP required to induce the same anti-apoptosis, antioxidation and anti-inflammatory efficiency. Furthermore, combined treatment may reduce apoptosis of neurons following mechanical injury in vitro. Although both compounds have each been extensively studied $(8,26)$, to the best of our knowledge, the current study is the first to identify the neurological outcome from combining TIIA and MP treatment following ASCI. The viability and regenerative capacity of neurons in the CNS is supported by the effects of TIIA and MP combined treatment. This has practical and conceptual implications due to the anti-apoptosis, antioxidation and anti-inflammatory efficiency of TIIA and MP combined treatment.

The treatment of ASCI is a major challenge for physicians due to the complex and progressive nature of the disease. MP is one of the most investigated agents due to its neuroprotective potential and remains the only drug used worldwide to treat ASCI. Although MP is not FDA-approved for use following ASCI, it is commonly used in this setting based on the results of a number of randomized controlled trials; furthermore, the beneficial effect of high-dose MP was initially reported in a series of National Acute Spinal Cord Injury Studies (NASCIS) $(13,27)$. Following publication of the NASCIS trials, the regimen of these trials was rapidly adopted worldwide.
As well as the benefits of MP however, high-dose MP led to a number of side effects developing, including urinary tract infections, pneumonia, wound infections, hyperglycemia and gastrointestinal complications (16,28-31). Therefore, the identification of other pharmacological agents to combine with MP is required in order to enhance functional recovery and reduce the dose of MP required and thus, its side effects. A number of studies demonstrate that TIIA may protect the spinal cord tissues from ASCI due to its anti-inflammatory, anti-apoptotic and antioxidant functions $(8,17,20,25)$. The present study aimed to detect the efficiency of a combined treatment of TIIA and MP following ASCI. The major finding of the current study is that combined TIIA and MP has beneficial effects on contusion acute injury of the spinal cord in rats and mechanical scratch in neurons.

When the spinal cord is lacerated or macerated by a sharp penetrating force, or contused or compressed by a blunt force, it leads to neurological damage occurring in the spinal cord that is normally referred to as 'primary injury'. The primary injury refers to the loss of spinal cord integrity due to mechanical factors (10). The mechanical injury leads to a cascade of biological events, described as 'secondary injury' $(32,33)$, which occurs over a time course of min to weeks and leads to further neurological damage. Much of the damage that occurs in the spinal cord following traumatic injury is due to the secondary effects of a complex array of pathophysiological processes including ischemia, edema, inflammation, excitotoxicity and oxidative cell damage, which take part in a 


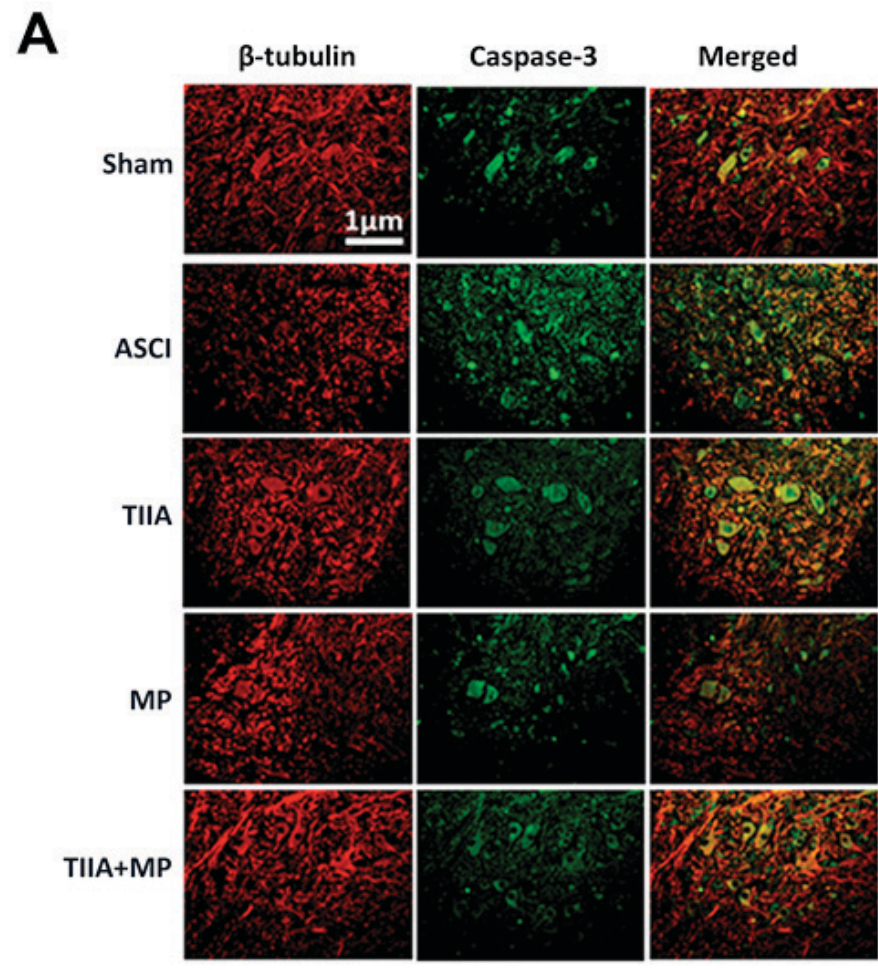

B

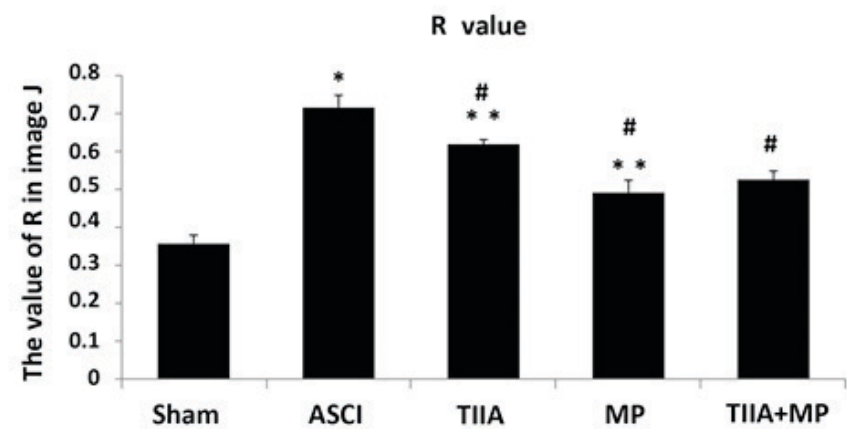

Figure 6. Immunofluorescence double labeling images and the $\mathrm{R}$ value of Mander's overlap coefficient indicate that the immunoreactivity of capase-3 increases in ASCI rats and was reduced following treatment with MP. Scale bar=1 $\mu \mathrm{M}$. (A) Expression of caspase-3 in the spinal tissues and neurons in vivo was determined by immunoreactivity at day 3 (red, $\beta$-III Tubulin and green, caspase-3). (B) Data are presented as mean \pm standard error of the mean, $\mathrm{n}=5$ per group. $\mathrm{R}$ value represented Mander's overlap coefficient tubulin and caspase-3 in ImageJ analysis software (v2.1.4.7): Ranges between 1 and 0 , with 1 indicating high colocalisation (increased levels of caspase- 3 in neurons) and 0 indicating low colocalisation (reduced levels of caspase- 3 in neurons). ${ }^{*} \mathrm{P}<0.05$ vs. Sham group; ${ }^{\text {"}} \mathrm{P}<0.05$ vs. ASCI group; ${ }^{* *} \mathrm{P}>0.05$ vs. TIIA + MP group. ASCI, acute spinal cord injury; MP, methylprednisolone; TIIA, tanshinone IIA; TIIA + MP, TIIA and MP combined treatment; Sham, sham-operated, no treatment.

spiraling interactive cascade, ending in neuronal dysfunction and death (10).

Assessment of neurological function is a prevalent method of accessing the degree of injury and the outcome of a treatment with medication. In the present study, BBB rating indicated a significant improvement of locomotion occurring everyday post-treatment, suggesting that the combined treatment may improve the behavioral function in animals with ASCI. Notably, the BBB scores in the MP group were higher than the combined treatment group 3 days following ASCI, while functional recovery was slower, taking 4-7 days. This may be because high-dose MP is more effective in protecting the injured spinal cord in the early phase, while continuous TIIA treatment may protect the injured spinal cord in the late phase.

The potential mechanism of ASCI remains unclear. However, neuronal apoptosis is one of the major pathogenic mechanisms that govern ASCI. The increased activation of oxidative stress and a series of apoptosis-associated proteins contribute to the pathogenic processes of neuronal death following ASCI, which ultimately lead to cell death $(4,7)$. Cell apoptosis typically occurs in the 'secondary injury' phase (32). $\mathrm{Bcl}-2$ and Bax are regarded as regulators of apoptosis, one of their principal roles is the regulation of the initiation of apoptosis by the mitochondrial pathway, and specifically, they directly regulate the permeability of the external mitochondrial membrane, acting in opposition: Bcl-2 inhibits apoptosis and Bax promotes apoptosis (34). The ratio of Bcl-2 to Bax controls the occurrence of apoptosis, whereby an increased ratio of Bcl-2 to Bax promotes cell survival and a decreased ratio promotes apoptosis (35). Caspases are a class of proteases instrumental in performing a number of cellular functions including cell differentiation, remodeling and death (36). 
Caspase-3 is a member of the caspase family, which regulates the execution of the mammalian apoptotic cell death program $(36,37)$. Caspase- 3 is the primary terminal in the process of cell apoptosis shear enzyme, an important mechanism of cell death following ASCI (4). Caspase-3 associated apoptotic pathways are activated following traumatic spinal cord injury in rats and occur early in neurons in the injury site and $h$ to days later in adjacent gliocytes, distant from the injury site. TIIA may protect damaged neurons and CNS tissues from apoptosis by regulating the expression of apoptosis factors, such as Bcl-2, Bax and caspase-3 (8,17,38-40).

In the present study, to investigate the protective effects of a combination treatment with TIIA and MP on the ASCI model, the factors associated with apoptosis were investigated. The data indicated that a combined treatment may markedly reverse the increase in pro-apoptosis factors Bax and caspase-3, and the decreased anti-apoptosis factor Bcl-2 protein induced by ASCI in vivo and in vitro. To the best of our knowledge, this is the first evidence that TIIA, combined with MP, may confer marked protection at a molecular level against ASCI in vivo. A previous study considered that MP may protect the oligodendrocytes but not neurons following spinal cord injury in vitro and in vivo (41). However, in the present study, the double-labeling immunofluorescence indicates that the expression of caspase-3(+)/ $\beta$-III Tubulin $(+)$ cells was significantly lower in the MP group and combined treatment group than in the ASCI group in vivo. These data demonstrated that combined treatment may protect neurons from apoptosis following ASCI with the same effectiveness as MP treatment alone.

Secondary injuries develop hours to days following primary injuries and involve a number of pathophysiological changes including ischemia, ion infiltration, production of oxygen free radicals and lipid peroxidation (7). The spinal cord consists largely of lipids and is easily damaged by free radical-induced lipid peroxidation, a major pathophysiological mechanism of secondary neuronal and glial damage (9). During this process, the balance between antioxidants and pro-oxidants is disrupted. For example, SOD, which is a scavenger for reactive oxygen species (ROS), is consumed during oxidative stress. MDA, formed from the breakdown of polyunsaturated fatty acids, serves as an important and reliable index for determining the extent of peroxidation reactions (42). Oxygen free radicals are typically produced in the mitochondria and their harmful effects are eliminated by antioxidant systems. Consistent with previous studies $(43,44)$, the results of the current study demonstrated that levels of MDA increased in the ASCI group and significantly decreased in the MP and combined treatment groups. However, no significant difference was observed between the expression of MDA between the combined treatment and MP groups on day 1 and 7. In addition, SOD levels also decreased in the ASCI group as compared with the sham group (data not shown) and the combined treatment and MP groups may increase the level of SOD in the blunt spinal cord injury tissue in the ASCI group.

$\mathrm{NF}-\kappa \mathrm{B}$ was key to the inflammatory action due to its regulatory role on the expression of a variety of cytokines (such as TNF- $\alpha$ and IL-6) that regulate the inflammatory response $(25,45)$. As a regulator of death and survival proteins, $\mathrm{NF}-\kappa \mathrm{B}$ serves an important role in neuron survival in the CNS.
A previous study revealed that $\mathrm{NF}-\kappa \mathrm{B}$ expression increased rapidly following the occurrence of ASCI, exhibiting an effect of enlarging the inflammatory cascade reaction and programmed cell death (26). The activation of $N F-\kappa B$ was closely linked to neuronal death from excitotoxicity and inhibiting NF- $\mathrm{NB}$ activation deterred the process of neural cell death. The inhibition of inflammatory responses at the early stage of ASCI may constitute an attractive therapeutic strategy for ASCI. Similar to previous studies $(46,47)$, the present study observed that the increase of $\mathrm{NF}-\kappa \mathrm{B}$ induced in contusion spinal cord was significantly reversed with the treatment of TIIA monotherapy, MP monotherapy and the combined treatment. However, there was no significant difference between the expression of $\mathrm{NF}-\kappa \mathrm{B}$ in the combined treatment and MP groups.

In conclusion, the present study demonstrates that TIIA combined with a low dose of MP may protect the spinal cord from ASCI due to its antioxidant, anti-inflammatory and anti-apoptosis properties in the spinal cord tissue following ASCI. The current study, to the best of our knowledge, is the first to reveal that TIIA combined with low dose MP therapy has the equivalent efficiency of a full dose of MP monotherapy in vivo and in vitro. Even though the present study has limitations, for example that the dosage of TIIA and MP in vivo was chosen artificially and the observation time was short, we believe that the combined treatment of TIIA and MP may be a promising strategy of pharmacological therapy for rapid initiation of neuroprotection following ASCI and reduce the dosage of MP in the treatment of ASCI for the same neuroprotective effect and further research should focus on exploring the biological and the long-term effects of combined treatment.

\section{Acknowledgements}

The authors would like to thank Dr. Hui Kang (Wuhan General Hospital of Guangzhou Military Command, Wuhan, China) for his advice about the experiment design and Professor Yi Zhang (Wuhan General Hospital of Guangzhou Military Command, Wuhan, China) for his technical assistance and his assistance with rat husbandry.

\section{References}

1. Singh A, Tetreault L, Kalsi-Ryan S, Nouri A and Fehlings MG: Global prevalence and incidence of traumatic spinal cord injury. Clin Epidemiol 6: 309-331, 2014.

2. Evaniew N, Belley-Côté EP, Fallah N, Noonan VK, Rivers CS and Dvorak MF: Methylprednisolone for the treatment of patients with acute spinal cord injuries: A systematic review and meta-analysis. J Neurotrauma 33: 468-481, 2016.

3. Choi DW and Rothman SM: The role of glutamate neurotoxicity in hypoxic-ischemic neuronal death. J Neurotrauma 13: 171-182, 1990.

4. Emery E, Aldana P, Bunge MB, Puckett W, Srinivasan A, Keane RW, Bethea J and Levi AD: Apoptosis after traumatic human spinal cord injury. J Neurosurg 89: 911-920, 1998.

5. Hall ED and Braughler JM: Free radicals in CNS injury. Res Publ Assoc Res Nerv Ment Dis 71: 81-105, 1993.

6. Blight AR: Macrophages and inflammatory damage in spinal cord injury. J Neurotrauma 9 (Suppl 1): S83-S91, 1992.

7. Lou J, Lenke LG, Ludwig FJ and O'Brien MF: Apoptosis as a mechanism of neuronal cell death following acute experimental spinal cord injury. Spinal Cord 36: 683-690, 1998.

8. Yin X, Yin Y, Cao FL, Chen YF, Peng Y, Hou WG, Sun SK and Luo ZJ: Tanshinone IIA attenuates the inflammatory response and apoptosis after traumatic injury of the spinal cord in adult rats. PLoS One 7: e38381, 2012. 
9. Silva NA, Sousa N, Reis RL and Salgado AJ: From basics to clinical: A comprehensive review on spinal cord injury. Prog Neurobiol 114: 25-57, 2014.

10. Kwon BK, Tetzlaff W, Grauer JN, Beiner J and Vaccaro AR: Pathophysiology and pharmacologic treatment of acute spinal cord injury. Spine J 4: 451-464, 2004.

11. Oh SK and Jeon SR: Current concept of stem cell therapy for spinal cord injury: A review. Korean J Neurotrauma 12: 40-46, 2016.

12. Bracken MB, Shepard MJ, Collins WF Jr, Holford TR, Baskin DS, Eisenberg HM, Flamm E, Leo-Summers L, Maroon JC, Marshall LF, et al: Methylprednisolone or naloxone treatment after acute spinal cord injury: 1-year follow-up data. Results of the second national acute spinal cord injury study. J Neurosurg 76: 23-31, 1992.

13. Bracken MB, Shepard MJ, Collins WF, Holford TR, Young W, Baskin DS, Eisenberg HM, Flamm E, Leo-Summers L, Maroon J, et al: A randomized, controlled trial of methylprednisolone or naloxone in the treatment of acute spinal-cord injury. Results of the second national acute spinal cord injury study. N Engl J Med 322: 1405-1411, 1990.

14. Bracken MB, Collins WF, Freeman DF, Shepard MJ, Wagner FW, Silten RM, Hellenbrand KG, Ransohoff J, Hunt WE Perot PL Jr, et al: Efficacy of methylprednisolone in acute spinal cord injury. JAMA 251: 45-52, 1984.

15. Harrop JS: Spinal cord injury: Debating the efficacy of methylprednisolone. Neurosurgery 61 (Suppl 1): S30-S31, 2014.

16. Walters BC, Hadley MN, Hurlbert RJ, Aarabi B, Dhall SS Gelb DE, Harrigan MR, Rozelle CJ, Ryken TC, Theodore N, et al: Guidelines for the management of acute cervical spine and spinal cord injuries: 2013 update. Neurosurgery 60 (Suppl 1): S82-S91, 2013.

17. Liu T, Jin H, Sun QR, Xu JH and Hu HT: The neuroprotective effects of tanshinone IIA on $\beta$-amyloid-induced toxicity in rat cortical neurons. Neuropharmacology 59: 595-604, 2010

18. Liu Z, Wang J, Huang E, Gao S, Li H, Lu J, Tian K, Little PJ Shen X, Xu S and Liu P: Tanshinone IIA suppresses cholesterol accumulation in human macrophages: Role of heme oxygenase-1. J Lipid Res 55: 201-213, 2014.

19. Yin Y, Sun W, Li Z, Zhang B, Cui H, Deng L, Xie P, Xiang J and Zou J: Effects of combining methylprednisolone with rolipram on functional recovery in adult rats following spinal cord injury. Neurochem Int 62: 903-912, 2013.

20. Kaech S and Banker G: Culturing hippocampal neurons. Nat Protoc 1: 2406-2415, 2006

21. Boomkamp SD, McGrath MA, Houslay MD and Barnett SC: Epac and the high affinity rolipram binding conformer of PDE4 modulate neurite outgrowth and myelination using an in vitro spinal cord injury model. Br J Pharmacol 171: 2385-2398, 2014.

22. Burnette WN: 'Western blotting': Electrophoretic transfer of proteins from sodium dodecyl sulfate-polyacrylamide gels to unmodified nitrocellulose and radiographic detection with antibody and radioiodinated protein A. Anal Biochem 112: 195-203, 1981.

23. Basso DM, Beattie MS and Bresnahan JC: A sensitive and reliable locomotor rating scale for open field testing in rats. J Neurotrauma 12: 1-21, 1995.

24. Tang Q, Han R, Xiao H, Shen J, Luo Q and Li J: Neuroprotective effects of tanshinone IIA and/or tetramethylpyrazine in cerebral ischemic injury in vivo and in vitro. Brain Res 1488: 81-91, 2012

25. Sun S, Yin Y, Yin X, Cao F, Luo D, Zhang T, Li Y and Ni L: Anti-nociceptive effects of Tanshinone IIA (TIIA) in a rat model of complete Freund's adjuvant (CFA)-induced inflammatory pain. Brain Res Bull 88: 581-588, 2012.

26. $\mathrm{Xu} \mathrm{J}$, Fan G, Chen $\mathrm{S}, \mathrm{Wu} Y, \mathrm{Xu} X M$ and $\mathrm{Hsu} C Y$ : Methylprednisolone inhibition of TNF-alpha expression and NF-kB activation after spinal cord injury in rats. Brain Res Mol Brain Res 59: 135-142, 1998.

27. A randomized, controlled trial of methylprednisolone or naloxone in the treatment of acute spinal-cord injury. N Engl J Med 323 1207-1209, 1990.

28. Ito Y, Sugimoto Y, Tomioka M, Kai N and Tanaka M: Does high dose methylprednisolone sodium succinate really improve neurological status in patient with acute cervical cord injury? A prospective study about neurological recovery and early complications. Spine (Phila Pa 1976) 34: 2121-2124, 2009.
29. Suberviola B, González-Castro A, Llorca J, Ortiz-Melón F and Miñambres E: Early complications of high-dose methylprednisolone in acute spinal cord injury patients. Injury 39: 748-752, 2008.

30. Matsumoto T, Tamaki T, Kawakami M, Yoshida M, Ando M and Yamada H: Early complications of high-dose methylprednisolone sodium succinate treatment in the follow-up of acute cervical spinal cord injury. Spine (Phila Pa 1976) 26: 426-430, 2001.

31. Chikuda H, Yasunaga H, Takeshita K, Horiguchi H, Kawaguchi H, Ohe K, Fushimi K and Tanaka S: Mortality and morbidity after high-dose methylprednisolone treatment in patients with acute cervical spinal cord injury: A propensity-matched analysis using a nationwide administrative database. Emerg Med J 31: 201-206, 2014.

32. Lu J, Ashwell KW and Waite P: Advances in secondary spinal cord injury: Role of apoptosis. Spine (Phila Pa 1976) 25: 1859-1866, 2000.

33. Mautes AE, Weinzierl MR, Donovan F and Noble LJ: Vascular events after spinal cord injury: Contribution to secondary pathogenesis. Phys Ther 80: 673-687, 2000.

34. Kroemer G, Galluzzi L and Brenner C: Mitochondrial membrane permeabilization in cell death. Physiol Rev 87: 99-163, 2007.

35. Korsmeyer SJ, Shutter JR, Veis DJ, Merry DE and Oltvai ZN: Bcl-2/Bax: A rheostat that regulates an anti-oxidant pathway and cell death. Semin Cancer Biol 4: 327-332, 1993.

36. Thornberry NA: The caspase family of cysteine proteases. $\mathrm{Br}$ Med Bull 53: 478-490, 1997.

37. Miller DK: The role of the Caspase family of cysteine proteases in apoptosis. Semin Immunol 9: 35-49, 1997.

38. Xia WJ, Yang M, Fok TF, Li K, Chan WY, Ng PC, Ng HK, Chik KW, Wang CC, Gu GJ, et al: Partial neuroprotective effect of pretreatment with tanshinone IIA on neonatal hypoxia-ischemia brain damage. Pediatr Res 58: 784-790, 2005.

39. Meng XF, Zou XJ, Peng B, Shi J, Guan XM and Zhang C: Inhibition of ethanol-induced toxicity by tanshinone IIA in PC12 cells. Acta Pharmacol Sin 27: 659-664, 2006.

40. Dong H, Mao S, Wei J, Liu B, Zhang Z, Zhang Q and Yan M: Tanshinone IIA protects PC12 cells from beta-amyloid(25-35)-induced apoptosis via PI3K/Akt signaling pathway. Mol Biol Rep 39: 6495-6503, 2012.

41. Lee JM, Yan P, Xiao Q, Chen S, Lee KY, Hsu CY and Xu J: Methylprednisolone protects oligodendrocytes but not neurons after spinal cord injury. J Neurosci 28: 3141-3149, 2008.

42. Ege E, Ilhan A, Gurel A, Akyol O and Ozen S: Erdosteine ameliorates neurological outcome and oxidative stress due to ischemia/reperfusion injury in rabbit spinal cord. Eur J Vasc Endovasc Surg 28: 379-386, 2004

43. Topsakal C, Erol FS, Ozveren MF, Yilmaz N and Ilhan N: Effects of methylprednisolone and dextromethorphan on lipid peroxidation in an experimental model of spinal cord injury. Neurosurg Rev 25: 258-266, 2002

44. Fu J, Huang H, Liu J, Pi R, Chen J and Liu P: Tanshinone IIA protects cardiac myocytes against oxidative stress-triggered damage and apoptosis. Eur J Pharmacol 568: 213-221, 2007.

45. Sadeghi A, Seyyed Ebrahimi SS, Golestani A and Meshkani R: Resveratrol Ameliorates Palmitate-Induced inflammation in skeletal muscle cells by attenuating oxidative stress and JNK/NF-KB pathway in a SIRT1-independent mechanism. J Cell Biochem Jan 6, 2017 (Epub ahead of print).

46. Chen Y, Wu X, Yu S, Lin X, Wu J, Li L, Zhao J and Zhao Y: Neuroprotection of tanshinone IIA against cerebral ischemia/reperfusion injury through inhibition of macrophage migration inhibitory factor in rats. PLoS One 7: e40165, 2012.

47. Chengke L, Weiwei L, Xiyang W, Ping W, Xiaoyang P, Zhengquan X, Hao Z, Penghui Z and Wei P: Effect of infliximab combined with methylprednisolone on expressions of NF- $\kappa \mathrm{B}$, TRADD, and FADD in rat acute spinal cord injury. Spine (Phila Pa 1976) 38: E861-E869, 2013. 